\title{
Probabilistic 4D Blood Flow Mapping
}

\author{
Ola Friman ${ }^{1}$, Anja Hennemuth ${ }^{1}$, Andreas Harloff ${ }^{2}$, Jelena Bock ${ }^{2}$, \\ Michael Markl ${ }^{2}$, and Heinz-Otto Peitgen ${ }^{1}$ \\ ${ }^{1}$ Fraunhofer MEVIS, Institute for Medical Image Computing, Germany \\ ${ }^{2}$ University Hospital Freiburg, Germany
}

\begin{abstract}
Blood flow and tissue velocity can be measured using phasecontrast MRI. In this work, the statistical properties of $4 \mathrm{D}$ phase-contrast images are derived, and a novel probabilistic blood flow mapping method based on sequential Monte Carlo sampling is presented. The resulting flow maps visualize and quantify the uncertainty in conventional flow visualization techniques such as streamlines and particle traces.
\end{abstract}

\section{Introduction}

Phase-contrast (PC) MRI utilizes residual phase shifts of spins to quantify tissue motion and blood flow [12. In cardiovascular PC MRI applications, visualization techniques, such as vector glyphs, streamlines, pathlines and particle traces, are employed for visualizing blood flow [3] . A 3D streamline illustrates the trajectory a zero-mass particle takes through a static vector field $\mathbb{V}(\mathbf{x}): \mathbb{R}^{3} \rightarrow \mathbb{R}^{3}$, e.g., an instantaneous flow field, see Fig. 4 a. Pathlines and particle traces show the particle trajectory in a vector field $\mathbb{V}(\mathbf{x}, t)$ that changes over time, e.g., in a $4 \mathrm{D}$ pulsative blood flow. Clinical applications that benefit from such flow pattern information include the assessment of stenoses, aneurysms, and heart valve function, the development of vessel plaque, and surgical planning and follow-up in congenital heart disease. While a visualization of the flow pattern using streamlines or particle traces indeed provide useful information, noise and uncertainty in the PC MRI measurements is not accounted for and the visualized traces may even give a false sense of precision. In this work, the uncertainty associated with a flow streamline or particle trace is addressed and visualized using the distribution of possible flow trajectories. To this end, the statistical properties of PC MRI images are first derived. The distribution of a streamline or a particle trace is then sampled and characterized using a sequential Monte Carlo approach.

\section{Statistical Properties of Flow MRI Images}

The statistical properties of PC MRI images in the presence of Gaussian measurement noise, particularly the statistical distribution of the estimated flow vectors in the estimated $4 \mathrm{D}$ flow vector field $\mathbb{V}(\mathbf{x}, t)$, are derived in this section. The derivation includes the use phased array MRI coils, which consist of a number of coils $K$ (typically 4-16) arranged in an array. Such arrays permit fast parallel imaging and/or a better Signal-to-Noise ratio (SNR) over large spatial extents [5]. The derivation below focusses on non-parallel imaging, which provides the highest image SNR.

T. Jiang et al. (Eds.): MICCAI 2010, Part III, LNCS 6363, pp. $416-423,2010$.
(c) Springer-Verlag Berlin Heidelberg 2010 

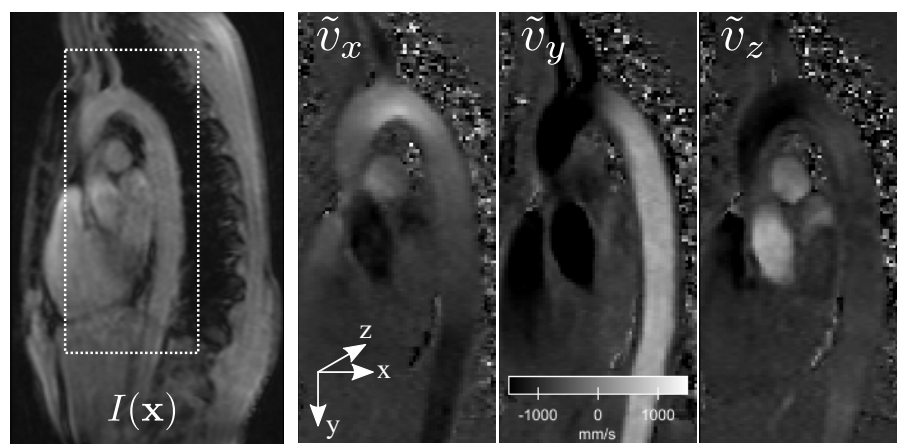

Fig. 1. Magnitude image $I(\mathbf{x})$ (left) and estimated velocity components $\tilde{v}_{x}, \tilde{v}_{y}$, and $\tilde{v}_{z}$ in the zoomed area around the aorta

\subsection{MRI Image Magnitude and Phase Modeling}

In general, a complex MRI image in the spatial domain acquired with coil $k$ in the phased array may be modeled as

$$
S_{k}(\mathbf{x})=A_{k}(\mathbf{x}) e^{i \theta_{k}(\mathbf{x})}+n_{k}^{r}(\mathbf{x})+i n_{k}^{i}(\mathbf{x})
$$

where $\mathbf{x} \in \mathbb{R}^{3}$ is a spatial voxel location, $A_{k}(\mathbf{x})$ represents the image magnitude weighted with the sensitivity profile of coil $k, \theta_{k}(\mathbf{x})$ is a coil-specific spatially varying phase, and $n_{k}^{r}(\mathbf{x})$ and $n_{k}^{i}(\mathbf{x})$ represent independent Gaussian noise $N\left(0, \sigma^{2}\right)$ in two quadrature channels [6]. From a statistical perspective, the magnitude image $\left|S_{k}(\mathbf{x})\right|$ follows a Rician probability distribution, which at high SNR $\left(A_{k} / \sigma>5\right)$ can be approximated by a Gaussian distribution [7] $\left|S_{k}(\mathbf{x})\right| \in N\left(A_{k}(\mathbf{x}), \sigma^{2}\right)$ for all practical purposes. This high-SNR assumption is generally true for voxels containing tissue or blood, which is shown in the Result section. A near-optimal-SNR magnitude image $I(\mathbf{x})$ can be reconstructed from all coils using the sum-of-squares method [5] (Fig. 1):

$$
I(\mathbf{x})=\sqrt{\sum_{k=1}^{K}\left|S_{k}(\mathbf{x})\right|^{2}} .
$$

The statistical properties of expressions such as the one in Eq. 2 have been studied in [8], and again, for high SNR voxels, a Gaussian approximation applies:

$$
I(\mathbf{x}) \in N\left(A(\mathbf{x}), \sigma^{2}\right), \text { where } A(\mathbf{x})=\sqrt{\sum_{k=1}^{K} A_{k}(\mathbf{x})^{2}} .
$$

Note that $A(\mathbf{x})$ and $\sigma^{2}$ are unknown, but with the knowledge of the statistical distribution in Eq. 3, they can be estimated from the known $I(\mathbf{x})$. This estimation is described in Section 2.3 . 
The phase $\theta_{k}(\mathbf{x})$ in Eq. 1 is estimated with the argument operator $\arg \left(S_{k}(\mathbf{x})\right)$, and the exact probability distribution of this estimator is given in [7]. For high SNR, however, one may again use a simpler Gaussian approximation

$$
\arg \left(S_{k}(\mathbf{x})\right) \in N_{w}\left(\theta_{k}(\mathbf{x}), \frac{\sigma^{2}}{A_{k}(\mathbf{x})^{2}}\right),
$$

where $N_{w}$ denotes the wrapped Normal distribution, i.e., $\theta_{k}$ is calculated modulo $2 \pi$ so that $\theta_{k} \in(-\pi, \pi]$.

\subsection{Flow Vector Modeling}

Four separate 3D PC MRI images must be acquired to reconstruct a 3D flow vector field $\mathbb{V}(\mathbf{x})$. A $4 \mathrm{D}$ flow field $\mathbb{V}(\mathbf{x}, t)$ is obtained by repeated acquisitions during the cardiac cycle synchronized with the RR-interval of the patient. The goal in this section is to derive the statistical properties of an estimated flow vector $\tilde{\mathbf{v}} \in \mathbb{V}(\mathbf{x}, t)$. All expressions in this section are applied voxelwise, and the spatial coordinate $\mathbf{x}$ is omitted for clarity. Typically, an encoding scheme is used which is based on a baseline image $S_{k}^{0}$ and three images $S_{k}^{x}, S_{k}^{y}$, and $S_{k}^{z}$ that encode the flow velocity along the $x, y$, and $z$ directions in the image phase, cf. Eq. 1.

$$
\begin{aligned}
S_{k}^{0} & =A_{k} e^{i \theta_{k}}+\text { noise, } & S_{k}^{y} & =A_{k} e^{i\left(\theta_{k}-\frac{\pi}{v_{\text {enc }}} v_{y}\right)}+\text { noise }, \\
S_{k}^{x} & =A_{k} e^{i\left(\theta_{k}-\frac{\pi}{v_{\text {enc }}} v_{x}\right)}+\text { noise }, & S_{k}^{z} & =A_{k} e^{i\left(\theta_{k}-\frac{\pi}{v_{\text {enc }}} v_{z}\right)}+\text { noise. }
\end{aligned}
$$

In Eq. 5, $v_{x}, v_{y}$, and $v_{z}$ are the true velocities along the orthogonal coordinate axes, and the velocity encoding parameter $v_{e n c}$ is a sequence parameter that controls the upper limit of the velocity that can be measured without artifacts. A typical value is $v_{e n c}=1.5 \mathrm{~m} / \mathrm{s} . \theta_{k}$ denotes an unknown and spatially varying phase for coil $k=1 \ldots K$, which is assumed constant over time and acquisitions. The flow velocity in the $x$-direction is found as the phase difference between the $S_{k}^{x}$ image and the baseline image $S_{k}^{0}$, i.e., following the sum-of-squares reconstruction in Eq. 2, a velocity estimate based on all $K$ coils is calculated as

$$
\tilde{v}_{x}=\frac{v_{\text {enc }}}{\pi} \arg \left(\sum_{k=1}^{K} S_{k}^{0} S_{k}^{x *}\right),
$$

where $S_{k}^{x *}$ denotes the complex conjugate of $S_{k}^{x}$. The estimates of $\tilde{v}_{y}$ and $\tilde{v}_{z}$ are calculated analogously. Example velocity images for one slice are shown in Fig. 1, Using Eq. 4, one can show that $\tilde{v}_{x}$ is distributed according to the following Gaussian distribution:

$$
\tilde{v}_{x} \in N\left(v_{x}, \frac{v_{e n c}^{2}}{\pi^{2}} \frac{2 \sigma^{2}}{A^{2}}\right),
$$

where $A$ is given in Eq. 3. It is assumed that the velocity is smaller than $v_{e n c}$ so that no phase wrap occurs. In practise, a standard image preprocessing step corrects such wraps. 
As each velocity component of the $3 \mathrm{D}$ flow vector $\tilde{\mathbf{v}}=\left[\tilde{v}_{x}, \tilde{v}_{y}, \tilde{v}_{z}\right]^{T}$ is Gaussian distributed according to Eq. 7, the joint distribution is multivariate Gaussian $\tilde{\mathbf{v}} \in N(\mathbf{v}, \mathbf{C})$. Furthermore, as the baseline images $S_{k}^{0}, k=1 \ldots K$ are involved in the computation of all three velocity components $\tilde{v}_{x}, \tilde{v}_{y}$, and $\tilde{v}_{z}$, the covariance matrix $\mathbf{C}$ will not be diagonal. For example, with a derivation similar to the one above, the covariance between $\tilde{v}_{x}$ and $\tilde{v}_{y}$ is

$$
\operatorname{Cov}\left(\tilde{v}_{x}, \tilde{v}_{y}\right)=\frac{v_{e n c}^{2}}{\pi^{2}} \frac{\sigma^{2}}{A^{2}}, \text { giving the full covariance } \mathbf{C}=\frac{v_{e n c}^{2}}{\pi^{2}} \frac{\sigma^{2}}{A^{2}}\left(\begin{array}{lll}
2 & 1 & 1 \\
1 & 2 & 1 \\
1 & 1 & 2
\end{array}\right) .
$$

To summarize, the measured flow velocity vector in each voxel may be see as drawn from a multivariate Gaussian distribution with mean v, i.e., the true velocity, and covariance matrix as given by Eq. 8. To fully specify the covariance matrix, estimates of the unknown parameters $A$ and $\sigma^{2}$ are required. This is discussed in the next section.

\subsection{Parameter Estimation}

Methods for estimating the noise variance $\sigma^{2}$ and signal strength $A_{k}$ from magnitude MRI images $\left|S_{k}\right|$ have previously been proposed in the literature 977]. Most of the proposed methods require a homogenous image region. Typically an air region is selected in which the SNR is low and the Gaussian approximation of the Rician distribution is no longer valid. In contrast, here we propose using all 4 image volumes in the PC MRI acquisition (cf. Eq. 5) to perform a voxelwise parameter estimation: Let $\Omega$ denote a mask of high-SNR voxels in the four images $I^{0}(\mathbf{x}), I^{x}(\mathbf{x}), I^{y}(\mathbf{x})$, and $I^{z}(\mathbf{x})$, reconstructed from Eq. 5 with the sum-of-squares method in Eq. 2. The high-SNR voxels are found using a straightforward image thresholding, as these voxels correspond to voxels with high intensity, i.e., all non-air voxels. For the voxels in $\Omega$, the Gaussian approximation in Eq. 3 can be applied, leading to an estimation procedure based on the well-known formulae for the mean and variance of Gaussian variables. First, an estimator of the signal strength $A(\mathbf{x})$ is obtained as

$$
\tilde{A}(\mathbf{x})=\frac{1}{4}\left[I^{0}(\mathbf{x})+I^{x}(\mathbf{x})+I^{y}(\mathbf{x})+I^{z}(\mathbf{x})\right] .
$$

According to Eq. 3, the noise variance $\sigma^{2}$ is equal for all voxels in $\Omega$, so that a variance estimate can first be calculated for each voxel separately, and a final estimate can then be found by pooling over all voxels:

$$
\tilde{\sigma}^{2}=\frac{1}{|\Omega|-1} \sum_{\mathbf{x}_{i} \in \Omega}\left[\frac{1}{4-1} \sum_{k=\{0, x, y, z\}}\left(I^{k}\left(\mathbf{x}_{i}\right)-\tilde{A}\left(\mathbf{x}_{i}\right)\right)^{2}\right] .
$$

In this expression, $|\Omega|$ denotes the number of voxels with high SNR. With $\tilde{A}(\mathbf{x})$ and $\tilde{\sigma}^{2}$, the covariance matrix for the flow vector in Eq. 8 is fully specified. 


\section{Probabilistic Flow Mapping}

In this section, the distribution of possible flow trajectories is addressed. Although there is no closed form expression of this distribution, it is possible to draw random samples using Monte Carlo methods and then reconstruct and visualize the distribution using a histogram technique.

\subsection{Probabilistic Streamlines and Particle Traces}

A 3D streamline is a trajectory described by train of vectors $\left\{\mathbf{s}_{0}, \mathbf{s}_{1}, \ldots, \mathbf{s}_{k-1}\right\}$ starting in a seed point $\mathbf{x}_{0} \in \mathbb{R}^{3}$, see Fig. 2. A 4D particle trace can be described similarly, although with the additional temporal dimension. Each vector $\mathbf{s}_{k}$ is a function of the current trajectory position $\mathbf{x}_{k}$, the flow vector field $\mathbb{V}$, and a step length parameter $T$ :

$$
\mathbf{s}_{k}=\mathbf{f}\left(\mathbf{x}_{k}, \mathbb{V} ; T\right) \text {. }
$$

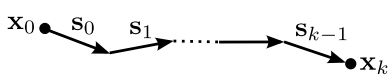

Fig. 2. A streamline

For example, an Euler sampling scheme uses $\mathbf{f}\left(\mathbf{x}_{k}, \mathbb{V} ; T\right)=T \mathbf{v}\left(\mathbf{x}_{k}\right)$, but more accurate multistage functions such as Heun's or 4 th-order Runge-Kutta schemes are generally used in practise [10]. The spatial position of the streamline trajectory after $k$ steps is

$$
\mathbf{x}_{k}=\mathbf{x}_{0}+\sum_{j=0}^{k-1} \mathbf{s}_{j} .
$$

In a conventional streamline algorithm, the vectors $\mathbf{s}_{k}$ are treated as deterministic variables. However, in PC MRI, these vectors are based on the estimated vector field $\mathbb{V}$, which is contaminated with random noise. Consequently, the vectors $\mathbf{s}_{k}$ as well as the positions $\mathbf{x}_{k}$ may be seen as random variables. The key question addressed in this work is the form of the statistical distribution $p\left(\mathbf{x}_{k}\right)=p\left(\mathbf{s}_{0}, \ldots, \mathbf{s}_{k-1}\right)$ of the streamline under the influence of noise in $\mathbb{V}$. A sample from $p\left(\mathbf{s}_{0}, \ldots, \mathbf{s}_{n-1}\right)$ is referred to here as a probabilistic streamline or a probabilistic particle trace. A theoretical derivation of this distribution is complicated by both the high dimensionality as well as the recursive nature of the streamline, i.e., the vector $\mathbf{s}_{k}$ depends on all previous vectors $\mathbf{s}_{k-1}, \ldots, \mathbf{s}_{0}$, which is clearly seen by combining Eq. 11 and Eq. 12. An alternative approach to investigate the form of a mathematically intractable probability distribution is to draw a large number of samples using computational Monte Carlo methods. An important observation is that the distribution above can be factorized into conditionally independent parts:

$$
p\left(\mathbf{s}_{0}, \ldots, \mathbf{s}_{k-1}\right)=\prod_{j=0}^{k-1} p_{j}\left(\mathbf{s}_{j} \mid \mathbf{x}_{j}\right) .
$$

Samples from such a distribution can be generated using so-called sequential Monte Carlo sampling [1]. In this sampling approach, a probabilistic streamline is generated iteratively by first drawing a sample $\tilde{\mathbf{s}}_{0}$ from $p_{0}\left(\mathbf{s}_{0} \mid \mathbf{x}_{0}\right)$, which 
gives $\tilde{\mathbf{x}}_{1}=\mathbf{x}_{0}+\tilde{\mathbf{s}}_{0}$. Next, given $\tilde{\mathbf{x}}_{1}$, a sample $\tilde{\mathbf{s}}_{1}$ can be drawn from $p_{1}\left(\mathbf{s}_{1} \mid \tilde{\mathbf{x}}_{1}\right)$, which gives $\tilde{\mathbf{x}}_{2}$, and so on. $p_{j}\left(\mathbf{s}_{j} \mid \mathbf{x}_{j}\right)$ remains to be determined in Eq. 13. Section 2.2 showed that the estimated flow vectors in $\mathbb{V}$ are Gaussian distributed with covariance matrix $\mathbf{C}$ in Eq. 8. Therefore, $p_{j}\left(\mathbf{s}_{j} \mid \mathbf{x}_{j}\right)$ also has a Gaussian form:

$$
p_{j}\left(\mathbf{s}_{j} \mid \mathbf{x}_{j}\right)=N\left[\mathbf{f}\left(\mathbf{x}_{j}, \mathbb{V} ; T\right), T^{2} \mathbf{C}\right] .
$$

Expressed in words, the random vector $\mathbf{s}_{j}$ has as mean the deterministic vector described by Eq.11]obtained using a regular streamline algorithm, e.g., an Euler, Heun or Runge-Kutta scheme. This vector is perturbed with the covariance matrix $\mathbf{C}$ in Eq. 8 scaled by the step length $T$.

\subsection{Probabilistic Flow Map}

Each probabilistic streamline or particle trace is a sample from the distribution $p\left(\mathbf{s}_{0}, \ldots, \mathbf{s}_{k-1}\right)$ that describes the probability of all possible paths a virtual zeromass particle may take from the seed point $\mathbf{x}_{0}$. To facilitate the visualization of this distribution, the probabilistic streamlines can be converted into a 3D spatial map $\Psi(\mathbf{x})$ or a 4 D spatiotemporal map $\Psi(\mathbf{x}, t)$ for particle traces. To this end, a large number $N>1000$ of probabilistic streamlines must be generated. Let $\mu(\mathbf{x}, N)$ be the number of occasions that each voxel $\mathbf{x}_{k}$ is passed by a streamline. The flow map is then calculated as a histogram where each voxel is a bin:

$$
\Psi(\mathbf{x})=\frac{\mu(\mathbf{x}, N)}{N} .
$$

A similar mapping technique has been used in Diffusion-Tensor MRI [12].

\section{Image Data}

Due to space limitations, a single 4D PC MRI data set of the blood flow in the aorta is used to demonstrate the proposed probabilistic blood flow mapping technique. The following acquisition parameters were used: $\mathrm{TE}=3.67 \mathrm{~ms}, \mathrm{TR}=$ $6.1 \mathrm{~ms}$, flip angle $15, v_{\text {enc }}=1.5 \mathrm{~m} / \mathrm{s}$, spatial resolution $1.7 \times 1.7 \times 3.5 \mathrm{~mm}^{3}$ and temporal resolution $48.8 \mathrm{~ms}$. A phased array coil with $K=12$ coil elements was used to obtain the necessary spatial coverage. The acquired $4 \mathrm{D}$ image volumes were of size $120 \times 192 \times 24$ voxels and 14 temporal frames covering a heart beat.

\section{Results}

To determine the covariance matrix of the flow vectors in Eq. 8, estimates of $A(\mathbf{x})$ and $\sigma^{2}$ were first calculated using the procedure described in Section 2.3 A voxelwise signal-to-noise image, where $\mathrm{SNR}=A(\mathbf{x}) / \sigma$, is shown in Fig. 3, The average SNR in the aorta is about 10, resulting in a standard deviation of $68 \mathrm{~mm} / \mathrm{s}$ in each velocity component when inserted into Eq. 7. As a reference, the peak flow velocity in the aorta is approximately $1500 \mathrm{~mm} / \mathrm{s}$ and significantly lower in smaller vessels. Moreover, the SNR is high enough to approximate the 


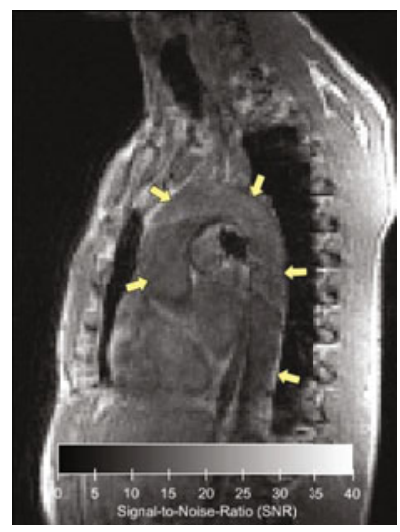

Fig. 3. SNR for each voxel in a slice estimated with the method presented in Section 2.3 The aorta is designated by the arrows.

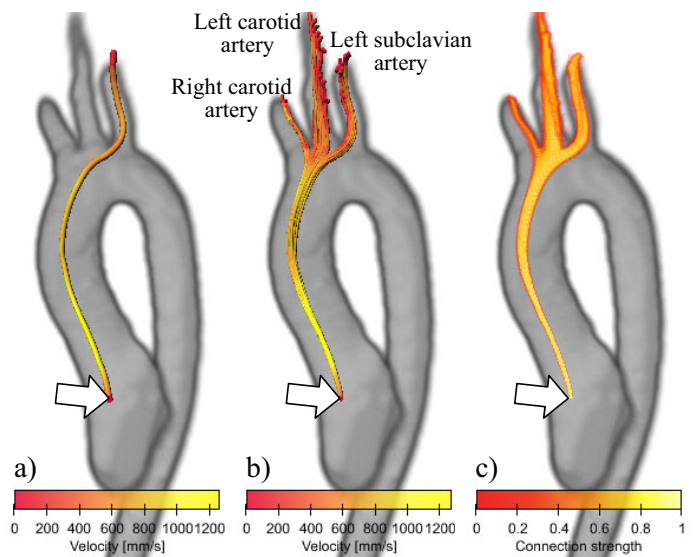

Fig. 4. a) A conventional $3 \mathrm{D}$ streamline visualizing the flow pattern from one point in the aorta. b) Probabilistic streamlines. c) Flow map $\Psi(\mathbf{x})$ calculated from 10,000 probabilistic streamlines.

Rician distribution with a Gaussian, as is done in Section 2, In Fig. 4h, a conventional streamline is shown, visualizing the flow pattern from the aorta into the left subclavian artery. In Fig. 4b, 50 probabilistic streamlines are shown, which were generated according to Section 3.1 and initiated in the same point. These probabilistic streamlines illustrate the uncertainty due to measurement noise that is not evident from the conventional streamline, i.e., when the noise is considered, the flow pattern may exit in any of the left carotid, right carotid, or left subclavian arteries. The distribution of possible flow trajectories, calculated as described in Section 3.2 using 10,000 probabilistic streamlines, is shown in Fig. 45. Figure 5 shows a 4D flow map generated from 10,000 probabilistic particle traces emitted from a region instead of from a single point. The color scale

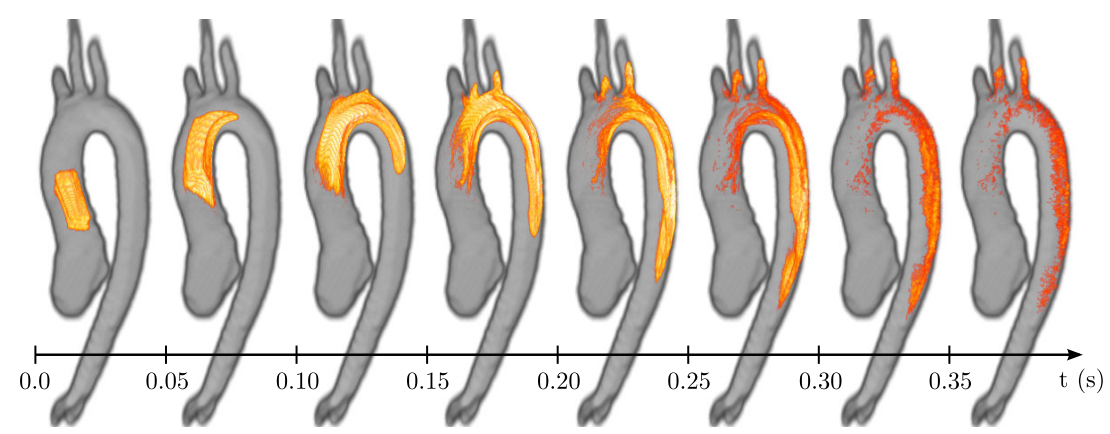

Fig. 5. 4D spatiotemporal probabilistic flow map $\Psi(\mathbf{x}, t)$ illustrating the statistical distribution of particle traces during a heart beat. The map is based on 10,000 probabilistic particle traces. 
represents the probability of finding a conventional particle trace in a specific spatiotemporal box when the measurement noise is considered.

\section{Discussion}

In this work, the statistical properties of PC MRI velocity measurements have been derived and used for mapping the uncertainty associated with blood flow streamlines and particle traces. This is done by drawing samples from the distribution of possible flow trajectories using a sequential Monte Carlo method. The primary application demonstrated here is a visualization of the uncertainty, i.e., the flow distribution. The next step is to employ the method for flow pattern quantification. For example, stroke embolization pathway probabilities can be calculated with a probabilistic flow connection map from a plaque position to critical vessels supplying the brain. Another concrete clinical application is congenital heart disease, where blood mixing ratios at positions where two vessels merge are of interest.

\section{References}

1. Pelc, N., Sommer, F., Li, K., Brosnan, T., Herfkens, R., Enzmann, D.: Quantitative magnetic resonance flow imaging. Magn. Reson. Q. 10(3), 125-147 (1994)

2. Markl, M., Harloff, A., Bley, T., Zaitsev, M., Jung, B., et al.: Time-resolved 3D MR velocity mapping at $3 \mathrm{~T}$ : Improved navigator-gated assessment of vascular anatomy and blood flow. J. Magn. Reson. Imaging 25(4), 824-831 (2007)

3. Napel, S., Lee, D., Frayne, R., Rutt, B.: Visualizing three-dimensional flow with simulated streamlines and three-dimensional phase-contrast MR imaging. J. Magn. Reson. Imaging 2(2), 143-153 (1992)

4. Wigström, L., Ebbers, T., Fyrenius, A., Karlsson, M., Engvall, J., Wranne, B., Bolger, A.: Particle trace visualization of intracardiac flow using time-resolved 3D phase contrast MRI. Magn. Reson. Med. 41(4), 793-799 (1999)

5. Roemer, P., Edelstein, W., Hayes, C., Souza, S., Mueller, O.: The NMR phased array. Magn. Reson. Med. 16(2), 192-225 (1990)

6. Conturo, T., Smith, G.: Signal-to-noise in phase angle reconstruction: dynamic range extension using phase reference offsets. Magn. Reson. Med. 15(3), 420-437 (1990)

7. Gudbjartsson, H., Patz, S.: The Rician distribution of noisy MRI data. Magn. Reson. Med. 34(6), 910-914 (1995)

8. Andersen, A.H., Kirsch, J.E.: Analysis of noise in phase contrast MR imaging. Med. Phys. 23(6), 857-869 (1996)

9. Henkelman, R.: Measurement of signal intensities in the presence of noise in MR images. Med. Phys. 12(2), 232-233 (1985)

10. Darmofal, D., Haimes, R.: An analysis of 3D particle path integration algorithms. J. Comput. Physics 123(1), 182-195 (1996)

11. Doucet, A., de Freitas, N., Gordon, N. (eds.): Sequential Monte Carlo Methods in Practice. Springer, New York (2001)

12. Koch, M., Norris, D., Hund-Georgiadis, M.: An investigation of functional and anatomical connectivity using magnetic resonance imaging. NeuroImage 16(1), 241-250 (2002) 